\title{
Haplotype-based association of regulator of G-protein signaling 5 gene polymorphisms with essential hypertension and metabolic parameters in Chinese
}

\author{
Bing Xiao ${ }^{1,2}$, Yi Zhang ${ }^{1,2,5}$, Wen-quan Niu ${ }^{1,2}$, \\ Pin-jing $\mathrm{Gao}^{1-4}$ and Ding-liang Zhu ${ }^{1-3,5, *}$ \\ ${ }^{1}$ State Key Laboratory of Medical Genomics, Ruijin \\ Hospital, Shanghai Jiaotong University School of \\ Medicine, Shanghai, P.R. China \\ ${ }^{2}$ Department of Cellular and Molecular Biology, \\ Shanghai Institute of Hypertension, Shanghai, \\ P.R. China \\ ${ }^{3}$ Shanghai Key Laboratory of Vascular Biology, \\ Shanghai, P.R. China \\ ${ }^{4}$ Laboratory of Vascular Biology, Institute of Health \\ Science Center, Shanghai Institutes for Biological \\ Sciences, Chinese Academy of Sciences, Shanghai, \\ P.R. China \\ ${ }^{5}$ Sino-French Research Center for Life Science and \\ Genomics, Shanghai, P.R. China
}

\begin{abstract}
Background: A recent genome-wide linkage study mapped blood pressure (BP)-related loci on human chromosome $1 \mathrm{q}$ and identified the regulator of G-protein signaling 5 (RGS5) as a candidate for regulation of BP. Thus, we assessed the relationship between RGS5 genetic polymorphisms and essential hypertension (EH) in Chinese.

Methods: A total of 906 patients with $\mathrm{EH}$ and 894 age- and gender-matched normotensive (NT) controls were enrolled. Sixteen single nucleotide polymorphisms (SNPs) in RGS5 were genotyped.

Results: There were no significant differences in the overall distributions of the genotypic and allelic frequencies for each SNPs between the two groups. However, in haplotype analysis, significant differences for the overall distributions were noted for four haplotypes constructed by five SNPs (rs12041294C/T, rs10917690A/G, rs10917695T/C, rs10917696T/C and rs2662774G/A), viz. $H_{2}(C-A-C-T-A)(p=0.038), H_{5}$ $(C-G-T-T-G)(p=0.001), H_{6}(T-G-C-T-A)(p=0.021)$ and $\mathrm{H}_{12}(\mathrm{~T}-\mathrm{A}-\mathrm{T}-\mathrm{T}-\mathrm{G})(\mathrm{p}=0.023)$. Serum concentrations of high- and low-density lipoprotein cholesterol showed significant associations with haplotypes revealed by a global test ( $p=0.0001$ and 0.0309 ).

Conclusions: Multiple SNPs in combination in RGS5 may confer risk for hypertension. Our results also

\footnotetext{
*Corresponding author: Prof. Ding-liang Zhu, Shanghai Institute of Hypertension, 197 Ruijin 2nd Road, Shanghai 200025, P.R. China

Phone/Fax: + 862154654498 ,

E-mail: zhudingliang@sibs.ac.cn previously published online October 28, 2009
}

Received April 23, 2009; accepted September 3, 2009;
\end{abstract}

lend support for the effect of RGS5 SNPs on lipid metabolism. Further studies are warranted to find the causal SNPs in RGS5 for EH.

Clin Chem Lab Med 2009;47:1483-8.

Keywords: haplotypes; hypertension; polymorphism; RGS5.

\section{Introduction}

Essential hypertension (EH) is a complex polygenic disease determined by both genetic and environmental factors (1). Genetic factors play an important role in determining susceptibility for EH. Elucidation of genetic variants contributing to an individual's susceptibility is important, but remains elusive due primarly to the heterogeneitic and polygenic nature of EH. Genetic variants have been shown to be associated with $\mathrm{EH}$ or blood pressure (BP), and thus warrants further investigation among different populations.

The regulator of G-protein signaling (protein: RGS and gene: $R G S$ ) are proteins modulating the Gprotein-coupled receptor (GPCR), induced signaling by enhancing hydrolysis of guanosine triphosphate (GTP). RGS plays a critical role in cardiovascular signal transduction. RGS proteins form a superfamily containing at least 25 members; each RGS is encoded by a different gene with different function (2-5). In the cardiovascular system, regulator of G-protein signaling 5 (RGS5) is an important signaling regulator. The physiological and pathological functions of RGS5 have been studied recently (6-8). A genome-wide linkage and candidate association study has identified a relation between human $R G S 5$ and increased BP in an American population (9). The expression of RGS5 was downregulated in cerebral microcapillaries of stroke-prone spontaneously hypertensive rats (10), and in resistant arteries taken from two animal models of hypertension (11). In addition, a RGS5 knockout mouse model displayed lower BP (12). These lines of evidence suggest the involvement of RGS5 in hypertension with an unknown mechanism. However, a literature search did not find any recent reports that assess the contribution of RGS5 variations to susceptibility of Chinese to hypertension. Therefore, we investigated the relationship of RGS5 variations with hypertension and intermediate phenotype in a large group of Han Chinese. 


\section{Study population and methods}

\section{Study population}

All subjects in this study were of Han Chinese descent and currently residing in the Shanghai area. A total of 1800 unrelated subjects were recruited, including 906 patients with $\mathrm{EH}$ and 894 normotensive (NT) controls. For each subject, clinical history and biochemical data were recorded. The study protocol was reviewed and approved by the Ethics Committee of Shanghai Ruijin Hospital, and written informed consent was obtained from each subject.

BP was measured by trained and certified hospital nurses using a mercury sphygmomanometer, according to a standard protocol recommended by the American Heart Association (13). BP was measured three times after rest in the seated position for at least $10 \mathrm{~min}$ and before breakfast in the morning. The mean of the three readings was used. Hypertension was defined as systolic blood pressure (SBP) $\geq 140 \mathrm{~mm} \mathrm{Hg}$ or diastolic blood pressure (DBP) $\geq 90 \mathrm{~mm} \mathrm{Hg}$ on three separate days, or the current use of antihypertensive drugs. In all patients, the onset of hypertension was before 70 years of age and secondary causes were ruled out through extensive clinical and biochemical studies. All NT controls were matched with $\mathrm{EH}$ patients for age, gender and area, with a SBP $<130 \mathrm{~mm} \mathrm{Hg}$ and DBP of $<85 \mathrm{~mm} \mathrm{Hg}$. They did not have a history of familial hypertension, hepatic disease, renal insufficiency and diabetes.

\section{Phenotype measurements}

Body mass index (BMI) was calculated from body weight and height, with weight in kilograms divided by the square of height in meters. Height and weight was recorded without shoes and with subjects wearing light indoor clothing. Plasma triglyceride (TG), total cholesterol (TC), and high-density lipoprotein cholesterol (HDL-C) concentrations were determined enzymatically using available kits and an auto analyzer in Ruijin Hospital. Concentrations of low-density lipoprotein cholesterol (LDL-C) were estimated using the Friedewald equation (14).

\section{Selection of single nucleotide polymorphisms (SNPs)}

Haplotype Mapping Project data (www.hapmap.org) tagger, developed by de Bakker et al. (15), was used for selection of tag SNP. Sixteen tag SNPs in RGS5 with minor allele frequencies of $>5 \%$ in the Han Chinese (Beijing, China) population were selected to capture the common variation in or around this gene, with a minimum $r^{2}$ of 0.80 . The information of the 16 tag SNPs in RGS5 is shown in Table 1.

\section{Genotyping}

Genomic DNA was extracted from leukocytes in peripheral blood sample using a standard phenol-chloroform method. All genotyping was performed using TaqMan assays and followed by allelic discrimination with the ABI Prism 7900HT Sequence Detection System (Applied Biosystems, Foster City, CA, USA). The RGS5 SNP Taqman probes and primers were obtained from Applied Biosystems Assay-by-Design Service for SNP genotyping. The Universal PCR Master Mix from Applied Biosystems was used in a $5-\mu L$ total reaction volume with $10 \mathrm{ng}$ DNA per reaction. Five percent of all genotypes were repeated in independent PCR reactions to check for consistency and to ensure intra- and inter-plate genotype quality control. On average, genotyping was successfully completed in $>97 \%$ subjects.

\section{Statistical analysis}

Hardy-Weinberg equilibrium (HWE) was assessed in each group using the $\chi^{2}$-test. Allele and genotype frequencies for each SNP comparing hypertensive and NT individuals were completed using SHEsis (16) software, available at http:// analysis.bio-x.cn/myAnalysis.php. For quantitative phenotypes, an ANOVA F test was used to examine the association between a genotype and phenotype. A two sided probability of $p \leq 0.05$ was considered statistically significant, unless otherwise indicated. The degree of linkage disequilibrium (LD) between SNPs within a gene was performed with Haplo view 4.1 software available at www.broad.mit. edu/mpg/haploview. Multifactor dimensionality reduction (MDR) software package (v.2.0.0), available at http://www. epistasis.org, was used to evaluate the interaction information of all SNPs tested with $\mathrm{D}^{\prime} \leq 0.8$, and to select the five best SNPs with strong synergism. To estimate haplotype frequencies in each group and test for association with phenotypes and $\mathrm{EH}$, we employed haplo.score and haplo. glm functions implemented in the Haplo.stats program (17, 18) (www.r-project.org). In addition, multiple-test was adjusted by using the false discovery rate (FDR) method with an

Table 1 Description of the selected SNPs in RGS5.

\begin{tabular}{|c|c|c|c|c|}
\hline SNP & Contig position & Region & Allele ${ }^{a}$ & $M A F^{b}$ \\
\hline rs1056515 & 161379884 & $3^{\prime}$ UTR & $\mathrm{G} / \mathrm{T}$ & 0.480 \\
\hline rs3806366 & 161381945 & 3' UTR & $A / G$ & 0.253 \\
\hline rs2841997 & 161386863 & Intron4 & $\mathrm{G} / \mathrm{A}$ & 0.239 \\
\hline rs16849973 & 161399331 & Intron2 & $A / G$ & 0.268 \\
\hline rs1876451 & 161399427 & Intron2 & $\mathrm{C} / \mathrm{T}$ & 0.170 \\
\hline rs6704267 & 161401028 & Intron2 & $A / C$ & 0.487 \\
\hline rs12029882 & 161406986 & Intron1 & $A / G$ & 0.169 \\
\hline rs12041294 & 161408115 & Intron1 & $\mathrm{C} / \mathrm{T}$ & 0.442 \\
\hline rs10917690 & 161408792 & Intron1 & $A / G$ & 0.476 \\
\hline rs12035879 & 161409179 & Intron1 & $\mathrm{G} / \mathrm{A}$ & 0.444 \\
\hline rs10917695 & 161415352 & Intron1 & $\mathrm{T} / \mathrm{C}$ & 0.336 \\
\hline rs10917696 & 161415949 & Intron1 & $\mathrm{T} / \mathrm{C}$ & 0.087 \\
\hline rs7528044 & 161416433 & Intron1 & $A / C$ & 0.157 \\
\hline rs4657247 & 161418521 & Intron1 & $\mathrm{C} / \mathrm{T}$ & 0.210 \\
\hline rs10799902 & 161426306 & Intron1 & $\mathrm{G} / \mathrm{A}$ & 0.092 \\
\hline rs2662774 & 161427644 & Intron1 & $\mathrm{G} / \mathrm{A}$ & 0.081 \\
\hline
\end{tabular}

UTR, untranslated region; MAF, minor allele frequency; ${ }^{a}$ major allele/minor allele; ${ }^{b}$ minor allele frequency in controls. 
FDR q-value threshold of 0.20 as suggested by Smith et al. (19).

\section{Results}

The baseline characteristics of the study population are shown in Table 2. There were no significant differences in age, gender, and serum concentrations of TC and LDL-C between patients with $\mathrm{EH}$ and NT controls.

\section{Single-point association analysis}

There were no deviations from HWE for all studied polymorphisms in controls $(p>0.05)$. As shown in Supplementary Table 1, no significant differences were noted in the distributions of the genotypic and allelic frequencies for all 16 SNPs between patients with $\mathrm{EH}$ and NT controls.

\section{Haplotype analysis}

For adjacent SNPs in strong LD $\left(D^{\prime}>0.8\right)$, we chose only one SNP for subsequent analyses with the MDR program. This was used to generate all possible combinations with genetic interaction. In this study, the Tuned Relief filter function was employed to select the top-five potential SNPs with strong synergism including rs12041294 C/T, rs10917690 A/G, rs10917695 T/C, rs10917696 T/C and rs2662774 G/A. These had a testing accuracy of 0.5572 and cross-validation consistency of 10 out of 10 . Table 3 shows the results of the haplotype analysis evaluated by the Haplo.score and Haplo.glm modules. Individual analysis identified significant associations of four haplotypes, $\mathrm{H}_{2}$ $(C-A-C-T-A)(p=0.038), H_{5}(C-G-T-T-G)(p=0.001)$, $\mathrm{H}_{6}(\mathrm{~T}-\mathrm{G}-\mathrm{C}-\mathrm{T}-\mathrm{A}) \quad(\mathrm{p}=0.021)$ and $\mathrm{H}_{12} \quad(\mathrm{~T}-\mathrm{A}-\mathrm{T}-\mathrm{T}-\mathrm{G})$ $(p=0.023)$, with $\mathrm{EH}$. Association results for haplotypes seen in $\mathrm{H}_{5}, \mathrm{H}_{6}$ and $\mathrm{H}_{12}$ had an independent effect on decreasing risk for hypertension after adjustment for age, gender and BMI, with an adjusted odds ratios of 0.357 for $\mathrm{H}_{5}(95 \% \mathrm{Cl}: 0.194-0.655), 0.329$ for $\mathrm{H}_{6}(95 \%$ Cl: $0.129-0.842)$ and 0.346 for $\mathrm{H}_{12}(95 \% \mathrm{Cl}: 0.138-$ $0.864)$, respectively. $\mathrm{H}_{2}$ had an independent effect on increasing risk for $\mathrm{EH}$ after adjusting for confounding factors, with an adjusted odds ratio of $1.727(95 \% \mathrm{Cl}$ : 1.029-2.897). Even after FDR adjustment for multiple testing, these associations remained significant, with FDR q-values less than the suggested threshold 0.20.

\section{Association for the RGS5 SNPs and metabolic parameters in NT controls}

The phenotype-genotype association was analyzed in NT controls only, since some clinical examinations were likely affected by drug treatment in patients with

Table 2 Demographics of the study participants.

\begin{tabular}{|c|c|c|c|}
\hline Variables & $\begin{array}{l}\text { Hypertensive } \\
\text { patients } \\
(\mathrm{n}=906)\end{array}$ & $\begin{array}{l}\text { Normotensive } \\
\text { controls } \\
(\mathrm{n}=894)\end{array}$ & p-Value \\
\hline Gender (men/women) & $454 / 452$ & $440 / 454$ & 0.705 \\
\hline Age, years & $51.7 \pm 8.9$ & $51.3 \pm 6.9$ & 0.331 \\
\hline $\mathrm{BMI}, \mathrm{kg} / \mathrm{m}^{2}$ & $25.9 \pm 4.2$ & $23.1 \pm 2.9$ & $<0.0001$ \\
\hline $\mathrm{SBP}, \mathrm{mm} \mathrm{Hg}$ & $149.9 \pm 21.5$ & $112.8 \pm 10.0$ & $<0.0001$ \\
\hline $\mathrm{DBP}, \mathrm{mm} \mathrm{Hg}$ & $93.6 \pm 13.5$ & $75.2 \pm 6.8$ & $<0.0001$ \\
\hline $\mathrm{TC}, \mathrm{mmol} / \mathrm{L}$ & $4.89 \pm 0.93$ & $4.83 \pm 0.95$ & 0.181 \\
\hline $\mathrm{TG}, \mathrm{mmol} / \mathrm{L}$ & $2.05 \pm 1.23$ & $1.27 \pm 0.77$ & $<0.0001$ \\
\hline LDL-C, mmol/L & $3.18 \pm 0.86$ & $3.18 \pm 0.92$ & 0.984 \\
\hline $\mathrm{HDL}-\mathrm{C}, \mathrm{mmo} / \mathrm{L}$ & $1.32 \pm 0.51$ & $1.48 \pm 0.33$ & $<0.0001$ \\
\hline
\end{tabular}

Data are expressed as mean \pm SD. BMI, body mass index; SBP, systolic blood pressure; DBP, diastolic blood pressure; TC, total cholesterol; TG, triglyceride; HDL-C, high-density lipoprotein cholesterol; LDL-C, low-density lipoprotein cholesterol.

Table 3 Distribution of haplotypes in patients with EH and NT subjects.

\begin{tabular}{|c|c|c|c|c|c|}
\hline Haplotype $^{a}$ & $\mathrm{EH}$ & NT & $p-$ Value $^{\mathrm{c}}$ & FDR q & OR $[95 \% \mathrm{Cl}]^{\mathrm{c}}$ \\
\hline $\mathrm{H}_{1} \mathrm{C}-\mathrm{A}-\mathrm{C}-\mathrm{C}-\mathrm{G}$ & 0.045 & 0.058 & 0.312 & 0.490 & $0.766[0.458-1.283]$ \\
\hline $\mathrm{H}_{2} \mathrm{C}-\mathrm{A}-\mathrm{C}-\mathrm{T}-\mathrm{A}$ & 0.048 & 0.028 & 0.038 & 0.106 & $1.727[1.029-2.897]$ \\
\hline $\mathrm{H}_{3} \mathrm{C}-\mathrm{A}-\mathrm{C}-\mathrm{T}-\mathrm{G}$ & 0.114 & 0.095 & 0.737 & 0.737 & $0.934[0.629-1.388]$ \\
\hline $\mathrm{H}_{4} \mathrm{C}-\mathrm{A}-\mathrm{T}-\mathrm{T}-\mathrm{G}$ & 0.287 & 0.261 & 0.353 & 0.485 & $0.873[0.656-1.162]$ \\
\hline $\mathrm{H}_{5} \mathrm{C}-\mathrm{G}-\mathrm{T}-\mathrm{T}-\mathrm{G}$ & 0.034 & 0.076 & 0.001 & 0.011 & $0.357[0.194-0.655]$ \\
\hline $\mathrm{H}_{6} \mathrm{~T}-\mathrm{G}-\mathrm{C}-\mathrm{T}-\mathrm{A}$ & 0.013 & 0.036 & 0.021 & 0.231 & $0.329[0.129-0.842]$ \\
\hline $\mathrm{H}_{7} \mathrm{~T}-\mathrm{G}-\mathrm{C}-\mathrm{T}-\mathrm{G}$ & 0.066 & 0.046 & 0.617 & 0.754 & $0.865[0.490-1.527]$ \\
\hline $\mathrm{H}_{9} \mathrm{C}-\mathrm{G}-\mathrm{C}-\mathrm{T}-\mathrm{G}$ & 0.015 & 0.013 & 0.662 & 0.728 & $0.797[0.289-2.201]$ \\
\hline $\mathrm{H}_{10} \mathrm{~T}-\mathrm{G}-\mathrm{C}-\mathrm{C}-\mathrm{G}$ & 0.026 & 0.015 & 0.272 & 0.499 & $1.626[0.684-3.867]$ \\
\hline $\mathrm{H}_{11} \mathrm{~T}-\mathrm{A}-\mathrm{C}-\mathrm{T}-\mathrm{G}$ & 0.010 & 0.016 & 0.134 & 0.295 & $0.299[0.061-1.453]$ \\
\hline $\mathrm{H}_{12} \mathrm{~T}-\mathrm{A}-\mathrm{T}-\mathrm{T}-\mathrm{G}$ & 0.008 & 0.050 & 0.023 & 0.084 & $0.346[0.138-0.864]$ \\
\hline $\mathrm{H}_{8} \mathrm{~T}-\mathrm{G}-\mathrm{T}-\mathrm{T}-\mathrm{G}^{\mathrm{b}}$ & 0.303 & 0.273 & & & BASE \\
\hline
\end{tabular}

${ }^{a}$ Combinational SNPs were arranged in the order as following: rs12041294 C/T, rs10917690 A/G, rs10917695 T/C, rs10917696 $\mathrm{T} / \mathrm{C}$ and rs2662774 G/A; ${ }^{\mathrm{b}} \mathrm{H}_{8} \mathrm{~T}-\mathrm{G}-\mathrm{T}-\mathrm{T}-\mathrm{G}$ was chosen as a reference haplotype in the analyses; ${ }^{\mathrm{c}} \mathrm{OR}[95 \% \mathrm{Cl}$; $\mathrm{p}-\mathrm{values}$ were calculated after adjustment for age, gender, BMI. 
$\mathrm{EH}$. We found that BMI was significantly higher for the rs12041294 minor group $\left(23.59 \pm 2.93 \mathrm{~kg} / \mathrm{m}^{2}\right)$ compared with the major $\left(22.90 \pm 3.15 \mathrm{~kg} / \mathrm{m}^{2}, \mathrm{p}=0.019\right)$ and heterozygous $\left(22.99 \pm 2.77 \mathrm{~kg} / \mathrm{m}^{2}, \quad p=0.027\right)$ groups. The serum concentration of TG was significantly higher for the rs2841997 major (1.29 $0.79 \mathrm{mmol} / \mathrm{L}, \mathrm{p}=0.025)$ and heterozygous (1.28 \pm $0.77 \mathrm{mmol} / \mathrm{L}, \mathrm{p}=0.035)$ groups compared with the minor group $(1.05 \pm 0.60 \mathrm{mmol} / \mathrm{L})$; the concentration of TC was significant higher for the rs2841997 heterozygous ( $4.81 \pm 0.96 \mathrm{mmol} / \mathrm{L}, \mathrm{p}=0.022$ ) group than the minor group ( $4.62 \pm 0.95 \mathrm{mmol} / \mathrm{L})$.

\section{Association for the RGS5 haplotypes and metabolic parameters in NT controls}

Table 4 lists the association of haplotypes with blood lipid concentrations. In NT controls, serum concentrations of HDL-C and LDL-C showed significant association with haplotypes (global $p=0.0001$ and 0.0309 ). Individual analysis found a significant association between $\mathrm{H}_{1}$ and HDL-C (Hap-score $=-2.87, \mathrm{p}=0.004$ ). Similar findings were seen in $\mathrm{H}_{3}$ (Hap-score $=2.48$, $p=0.013), H_{5} \quad$ (Hap-score $\left.=2.35, p=0.019\right), H_{6} \quad($ Hapscore $=-2.16, \mathrm{p}=0.031)$ and $\mathrm{H}_{12} \quad$ (Hap-score $=2.58$, $\mathrm{p}=0.01$ ). For $\mathrm{H}_{12}$, there was a significant association with TC (Hap-score $=2.40, \mathrm{p}=0.016$ ) and LDL-C (Hapscore $=2.97, p=0.003$ ). In addition, significant associations between $\mathrm{H}_{11}$ and SBP (Hap-score $=2.09$, $\mathrm{p}=0.037$ ), $\mathrm{H}_{3}$ and DBP (Hap-score $=-2.02, \mathrm{p}=0.043$ ) were found.

\section{Discussion}

The candidate gene approach may not replace the genome-wide scan strategy in detecting the genetics of complex traits. However, it is an important alternative strategy, especially when the population studied is large enough, and the candidate genes are selected on the basis of strong biological hints (20). It has been proposed that to generate robust data, a much larger sample involving 1000 subjects in each group might be required (21). Our sample size included 906 cases and 894 controls. To the best of our knowledge, this is the first case-control study to date focusing on RGS5 polymorphisms and susceptibility to $\mathrm{EH}$ in Han Chinese.
A recent study documented that the 1q23-32 regions harbor multiple $\mathrm{EH}$-susceptible genes that affect BP, and provided evidence of an independent association between three genes in this region and BP. RGS5 was one of the three genes (9). In addition, several genome linkage studies of $\mathrm{BP}$ and $\mathrm{EH}$ in humans, as well as in rat and mouse models of hypertension, suggest that chromosome 1q23-q32 regions were likely to contain EH-susceptible loci (22-27). Along with these lines of evidence, we consider RGS5 as a candidate gene for investigating genetic susceptibility to $\mathrm{EH}$ in a large Han Chinese population. Chang et al. (9) investigated multiple genes with respect to susceptibility to EH and found that a total of 13 SNPs in RGS5 were assigned in two LD blocks. Therefore, eight SNPs showed significant associations with BP in at least one of six sample groups studied. In contrast, in the present study, all 16 SNPs covered these two LD blocks, but none showed any significant association with $\mathrm{EH}$ in single point analysis. In addition, some SNPs, such as rs3806366, showed great genetic heterogeneity. For example, the rs3806366 A allele frequency was 0.75 in this study. This is between that of European-Americans (0.91) and African-Americans (0.50) in the study of Chang et al. (9). In view of genetic heterogeneity among different ethnic populations, it would be important to construct a database of polymorphisms responsible for $\mathrm{EH}$ for each race or ethnic group.

As we previously indicated, haplotype analysis could provide more information about the effect of genetic interaction, especially when these alleles have a synergistic effect (28). Therefore, we constructed haplotype models using the six MDR derived polymorphisms as a whole. It is generally believed that haplotype analysis, which studies single genetic variant in their combination simultaneously, has a higher complexity level than single-locus analysis. Also, this has more power for assessing the association between candidate genes and complex diseases (29). This approach does not require the causal variants be identified or directly tested, but rather has the potential to highlight physical regions that harbor putative disease-associated variants (30). In this study, our haplotype-based association study revealed some predominance of SNP combinations associated with disease status, and conferred increased or decreased

Table 4 Blood lipid parameters by individual haplotypes.

\begin{tabular}{|c|c|c|c|c|c|c|c|c|}
\hline \multirow[t]{2}{*}{ Haplotype ${ }^{a}$} & \multicolumn{2}{|l|}{ TG } & \multicolumn{2}{|l|}{$\mathrm{TC}$} & \multicolumn{2}{|l|}{ HDL-C } & \multicolumn{2}{|l|}{ LDL-C } \\
\hline & Hap-score & $\mathrm{p}$-Value & Hap-score & p-Value & Hap-score & $p$-Value & Hap-score & p-Value \\
\hline $\mathrm{H}_{1}$ C-A-C-C-G & -1.62 & 0.106 & 1.57 & 0.117 & -2.87 & 0.004 & -1.35 & 0.179 \\
\hline $\mathrm{H}_{3}$ C-A-C-T-G & -0.42 & 0.674 & 1.43 & 0.153 & 2.48 & 0.013 & 1.22 & 0.221 \\
\hline $\mathrm{H}_{4}$ C-A-T-T-G & 1.89 & 0.059 & -1.91 & 0.056 & -1.58 & 0.115 & -0.63 & 0.528 \\
\hline $\mathrm{H}_{5}$ C-G-T-T-G & -0.21 & 0.831 & 1.11 & 0.269 & 2.35 & 0.019 & 1.08 & 0.278 \\
\hline $\mathrm{H}_{6} \mathrm{~T}-\mathrm{G}-\mathrm{C}-\mathrm{T}-\mathrm{A}$ & -1.62 & 0.106 & -0.74 & 0.458 & -2.16 & 0.031 & -1.89 & 0.059 \\
\hline $\mathrm{H}_{7}$ T-G-C-T-G & -0.60 & 0.547 & -1.18 & 0.239 & -0.58 & 0.564 & -1.65 & 0.099 \\
\hline $\mathrm{H}_{12}$ T-A-T-T-G & 0.06 & 0.950 & 2.40 & 0.016 & 2.58 & 0.010 & 2.97 & 0.003 \\
\hline $\mathrm{H}_{8}$ T-G-T-T-G & -0.55 & 0.582 & -0.26 & 0.791 & -1.67 & 0.095 & -1.33 & 0.184 \\
\hline
\end{tabular}

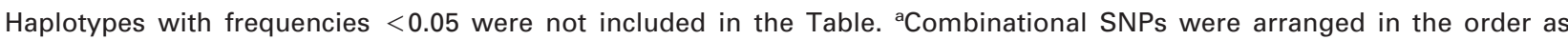
following: rs12041294 C/T, rs10917690 A/G, rs10917695 T/C, rs10917696 T/C and rs2662774 G/A. 
risk for EH. For example, haplotypes $\mathrm{H}_{5}(\mathrm{C}-\mathrm{G}-\mathrm{T}-\mathrm{T}-\mathrm{G})$, $\mathrm{H}_{6}(\mathrm{~T}-\mathrm{G}-\mathrm{C}-\mathrm{T}-\mathrm{A}), \mathrm{H}_{12}$ (T-A-T-T-G) had a $64.3 \%-$ $67.1 \%$ decreased risk for $\mathrm{EH}$, while $\mathrm{H}_{2}$ (C-A-C-T-A) had a $72.7 \%$ increased risk. If the polymorphisms derived by MDR function were actually involved, or were markers in strong linkage with other functional polymorphisms involved in the pathogenesis of $\mathrm{EH}$, two possible inferences can be drawn. One is that rs10917696 is unlikely to play a leading role since the $\mathrm{T}$ allele harbors both protective and risk-conferring haplotypes. Another inference is the genetic interaction between rs12041294 C/T and rs10917690 A/G polymorphisms, because haplotypes with one or two mutant allele(s), such as C-G, T-A, T-G in these two SNPs showed decreased risk, whereas haplotypes with two wild alleles (C-A) resulted in increased risk for EH. Because statistical interaction may not automatically imply biological interaction, haplotype analysis may represent the first step in providing clues for directing future research.

We performed phenotype-genotype associations among NT controls only. This is because these clinical phenotypes might be confounded by drug regimens in patients with $\mathrm{EH}$. Of note, individuals carrying the rs12041294 2/2 genotype had significantly higher BMI than those carrying the $1 / 1$ and $1 / 2$ genotype. Also, TG concentrations were significantly higher for individuals carrying the rs2841997 $1 / 1$ and $1 / 2$ genotype compared with those carrying the $2 / 2$ genotype. A similar trend was noted for TC. Expanding these findings, significant associations were also found between several haplotypes and blood lipids. Our results suggest that RGS5 might play a role in the control of body weight and lipid metabolisms, in agreement with previous studies. Cho et al. reported that Rgs5-/- mice weighed less than littermate controls. The relative fat content per gram of weight of the littermate controls was significantly higher than that of the Rgs5-/- mice (12). In addition, RGS5 has been reported to be expressed in mouse adipocytes isolated from subcutaneous and intra-abdominal fat. Given that the association analyses of our quantitative trait were based on the control group, and only limited SNPs showed marginally significant associations with lipid parameters, further studies will be required in the population at large to test its effect on body weight and lipid metabolism.

Despite the strengths of the present study, including the large sample size and systematic selection of RGS5 polymorphisms, our study should be interpreted within the context of its limitations. First, for the case-control nature of this study design, it inevitably suffers from the limitations of this type of study; i.e., the inability to prove the existence of a causality relationship. Second, we genotyped only 16 common polymorphisms in RGS5, and did not examine other genes/polymorphisms that might be associated with hypertension. Also, the polymorphisms selected do not cover the genes fully and extensively. Third, other risk factors or intermediate phenotypes, such as lifestyles (salt consumption and physical activity etc.) were unavailable for this analysis. Therefore, our results should be considered preliminary. In addition, confirmation from a biological or clinical point of view is critical.

In conclusion, the present study suggests multiple variants in combination in $R G S 5$, rather than a single SNP, may alter the risk for hypertension. Further studies are warranted to find the causal variations of RGS5 in $\mathrm{EH}$, and to prove the effect of RGS5 gene variants on lipid metabolism.

Supplementary data associated with this article can be found in the online version at: http://www.reference-global.com/ doi/suppl/10.1515/CCLM.2009.215.

\section{Conflict of interest statement}

We did not accept any funding or support from an organization that may in any way gain or lose financially from the results of our study. We have not been employed by an organization that may in any way gain or lose financially from the results of your study. We do not have any other conflicting interests.

\section{Acknowledgements}

This work was supported by the projects 973 and 863 of the Ministry of Science and Technology of China (grant numbers 2004CB518600, 2009CB521905 and 2006 AA 02Z179), the National Natural Science Foundation of China (grant number 30771184 and 30600243) and the Shanghai Commission of Science and Technology (grant number 08 DZ2200400 and 08410701900).

\section{References}

1. Luft FC. Geneticism of essential hypertension. Hypertension 2004;43:1155-9.

2. Hollinger S, Hepler JR. Cellular regulation of RGS proteins. Modulators and integrators of $\mathrm{G}$ protein signaling. Pharmacol Rev 2002;54:527-59.

3. Schieffer B, Drexler H, Ling BN, Marrero MB. G protein coupled receptors control vascular smooth muscle cell proliferation via pp60c-src and p21ras. Am J Physiol 1997;272:C2019-30.

4. Wieland T, Mittmann C. Regulators of G-protein signaling: multifunctional proteins with impact on signaling in the cardiovascular system. Pharmacol Ther 2003;97:95115.

5. Hao J, Michalek C, Zhang W, Zhu M, Xu X, Mende U. Regulation of cardiomyocyte signaling by RGS proteins: differential selectivity towards $\mathrm{G}$ proteins and susceptibility to regulation. J Mol Cell Cardiol 2006;41:51-61.

6. Cho H, Kozasa T, Bondjers C, Betsholtz C, Kehrl JH. Pericyte-specific expression of RGS5: implications for PDGF and EDG receptor signaling during vascular maturation. FASEB J 2003;17:440-2.

7. Li J, Adams LD, Wang X, Pabon L, Schwartz SM, Sane $D C$, et al. Regulator of $G$ protein signaling 5 marks peripheral arterial smooth muscle cells and is downregulated in atherosclerotic plaque. J Vasc Surg 2004;40:519-28.

8. Wang X, Adams LD, Pabón LM, Mahoney WM Jr, Beaudry D, Gunaje J. RGS5, RGS4, and RGS2 expression and aortic contractibility are dynamically co-regulated during aor- 
tic banding-induced hypertrophy. J Mol Cell Cardiol 2008;44:539-50.

9. Chang YP, Liu X, Kim JD, Ikeda MA, Layton MR, Weder $A B$. Multiple genes for essential-hypertension susceptibility on chromosome1q. Am J Hum Genet 2007;80:25364.

10. Kirsch T, Wellner M, Luft FC, Haller H, Lippoldt A. Altered gene expression in cerebral capillaries of stroke-prone spontaneously hypertensive rats. Brain Res 2001;910: 106-15.

11. Grayson TH, Ohms SJ, Brackenbury TD, Meaney KR, Peng K, Pittelkow YE, et al. Vascular microarray profiling in two models of hypertension identifies caveolin-1, Rgs2 and Rgs5 as antihypertensive targets. BMC Genomics 2007;8:404.

12. Cho H, Park C, Hwang IY, Han SB, Schimel D, Despres $\mathrm{D}$, et al. Rgs5 targeting leads to chronic low blood pressure and a lean body habitus. Mol Cell Biol 2008;28: 2590-7.

13. Perloff D, Grim C, Flack J, Frohlich ED, Hill M, McDonald $M$, et al. Human blood pressure determination by sphygmomanometry. Circulation 1993;88:2460-70.

14. Friedewald WT, Levy RI, Fredrickson DS. Estimation of the concentration of low-density lipoprotein cholesterol in plasma, without use of the preparative ultracentrifuge. Clin Chem 1972;18:499-502.

15. de Bakker PI, Yelensky R, Pe'er I, Gabriel SB, Daly MJ, Altshuler D. Efficiency and power in genetic association studies. Nat Genet 2005;37:1217-23.

16. Shi YY, He L. SHEsis, a powerful software platform for analyses of linkage disequilibrium, haplotype construction, and genetic association at polymorphism loci. Cell Res 2005;15:97-8.

17. Schaid DJ, Rowland CM, Tines DE, Jacobson RM, Poland GA. Score tests for association between traits and haplotypes when linkage phase is ambiguous. Am J Hum Genet 2002;70:425-34.

18. Lake SL, Lyon H, Tantisira K, Silverman EK, Weiss ST, Laird NM, et al. Estimation and tests of haplotype- environment interaction when linkage phase is ambiguous. Hum Hered 2003;55:56-65.

19. Smith NL, Hindorff LA, Heckbert SR, Lemaitre RN, Marciante KD, Rice K, et al. Association of genetic variations with nonfatal venous thrombosis in postmenopausal women. J Am Med Assoc 2007;297:489-98.

20. Tabor HK, Risch NJ, Myers RM. Candidate-gene approaches for studying complex genetic traits: practical considerations. Nat Rev Genet 2002;3:391-7.

21. Cardon LR, Bell JI. Association study designs for complex diseases. Nat Rev Genet 2001;2:91-9.

22. Perola $M$, Kainulainen $K$, Pajukanta $P$, Terwilliger JD, Hiekkalinna T, Ellonen P, et al. Genome-wide scan of predisposing loci for increased diastolic blood pressure in Finnish siblings. J Hypertens 2000;18:1579-85.

23. Hunt SC, Ellison RC, Atwood LD, Pankow JS, Province MA, Leppert MF. Genome scans for blood pressure and hypertension: the National Heart, Lung, and Blood Institute Family Heart Study Hypertension 2002;40:1-6.

24. James K, Weitzel LR, Engelman CD, Zerbe G, Norris JM. Genome scan linkage results for longitudinal systolic blood pressure phenotypes in subjects from the Framingham Heart Study. BMC Genet 2003;4:S83.

25. DiPetrillo K, Tsaih SW, Sheehan S, Johns C, Kelmenson $P$, Gavras H, et al. Genetic analysis of blood pressure in $\mathrm{C} 3 \mathrm{H} / \mathrm{HeJ}$ and SWR/J mice. Physiol Genomics 2004;17: 215-20.

26. Stoll M, Kwitek-Black AE, Cowley AW Jr, Harris EL, Harrap SB, Krieger JE, et al. New target regions for human hypertension via comparative genomics. Genome Res 2000;10:473-82.

27. Sugiyama F, Churchill GA, Higgins DC, Johns C, Makaritsis KP, Gavras $\mathrm{H}$, et al. Concordance of murine quantitative trait loci for salt-induced hypertension with rat and human loci. Genomics 2001;71:70-7.

28. Qi Y, Niu W, Zhou W, Hou S, Qiu C. Correlation between angiotensinogen gene polymorphisms and essential hypertension in Chinese population. J Hum Hypertens 2008;22:147-50.

29. Rioux JD, Daly MJ, Silverberg MS, Lindblad K, Steinhart $\mathrm{H}$, Cohen $\mathrm{Z}$, et al. Genetic variation in the $5 \mathrm{q} 31$ cytokine gene cluster confers susceptibility to Crohn disease. Nat Genet 2001;29:223-8.

30. Lu X, Zhao W, Huang J, Li H, Yang W, Wang L, et al. Common variation in KLKB1 and essential hypertension risk: tagging-SNP haplotype analysis in a case-control study. Hum Genet 2007;121:327-35. 